\title{
Celiac Disease in Children with Moderate-to-Severe Iron-deficiency Anemia
}

\author{
Manish Narang $^{1}$, Ravikumar Natarajan ${ }^{1}$, Dheeraj Shah ${ }^{1}$, Amarender Singh Puri ${ }^{2}$, Vikas Manchanda $^{3}$ \\ and Mrinalini Kotru ${ }^{4}$ \\ From ${ }^{1}$ Division of Pediatric Gastroenterology, Department of Pediatrics and ${ }^{4}$ Department of Pathology, University College of \\ Medical Sciences and GTB Hospital; ${ }^{2}$ Department of Gastroenterology, GB Pant Institute of Post Graduate Medical Education and \\ Research;and ${ }^{3}$ Department of Clinical Microbiology and Infectious Diseases, Chacha Nehru Bal Chikitsalaya; Delhi, India
}

Correspondence to: Dr Manish Narang, Professor, Department of Pediatrics, UCMS and GTB Hospital, Dilshad Garden, Delhi 110095, India. manish_2710@yahoo.com Received: December 28, 2016; Initial review: February 09, 2017; Accepted: September 22, 2017.
Objective: To evaluate the proportion of children with moderate to severe iron-deficiency anemia who have associated celiac disease. Methods: This cross-sectional analytical study was conducted among children aged 1 to 12 years of age with moderate-to-severe iron deficiency anemia and control children without anemia.Serum IgA-tissue trans-glutaminase levels were assessed in both cases and controls. All children with positive celiac serology underwent upper gastrointestinal endoscopy and duodenal biopsy; biopsy finding of Marsh grade 3 was considered positive for celiac disease. Results: There were 152 anemic children and 152 controls with mean (SD) hemoglobinof $7.7(1.8)$ and $12.2(0.74) \mathrm{g} / \mathrm{dL}$, respectively. $16(10.5 \%)$ cases and $3(2 \%)$ control patients had positive serology for celiac disease [OR $(95 \% \mathrm{Cl}) 5.33(1.52-18.67), P=0.007]$. Six $(3.9 \%)$ children with iron-deficiency anemia and none of the controls had biopsy features diagnostic of celiac disease. Conclusion:In the Northern Indian tertiary-care hospital outpatient setting, Celiac disease was associated with $4 \%$ of children presenting with moderate-to-severe anemia.

Keywords: Biopsy, Diagnosis, Endoscopy, Transglutaminases.
U nexplained iron-deficiency anemia without gastro-intestinal symptoms is a wellrecognized presentation of celiac disease (CD) [1]. However, the data regarding the proportional contribution of $\mathrm{CD}$ to unexplained anemia in children are scarce, especially from a country like India where nutritional anemias are very common [2]. Children diagnosed with $\mathrm{CD}$ require major dietary modifications in addition to the treatment of the nutritional anemia [3]. This study was conducted to assess proportionate contribution of celiac disease as the underlying causative factor of the iron deficiency anemia.

\section{MethodS}

This cross-sectional study was conducted in the pediatric outpatient department of a tertiary-care center in Delhi over a period of 18 months in 2013-15. Children between the ages of 1 and 12 years with visible pallor as detected on physical examination were included in the study if they had moderate-to-severe iron-deficiency anemia (IDA). Moderate anemia was defined as hemoglobin concentration: $7-9.9 \mathrm{~g} / \mathrm{dL}$ in children $<5$ years of age and $8-10.9 \mathrm{~g} / \mathrm{dL}$ in children of 5 to 12 years of age. Severe anemia was defined as hemoglobin concentration: $<7 \mathrm{~g} / \mathrm{dL}$ in children $<5$ years of age; $<8 \mathrm{~g} / \mathrm{dL}$ in children of 5 to 12 years of age [4]. Iron-deficiency was considered as a cause of anemia if serum ferritin level was $<12 \mathrm{ng} / \mathrm{mL}$ and/or transferrin saturation $<16 \%[5,6]$. Healthy siblings of patients visiting the outpatient department were chosen as controls. The controls consisted of children with normal

Accompanying Editorial: Pages 23-24.

hemoglobin level as per the age (hemoglobin $>11 \mathrm{~g} / \mathrm{dL}$ in $12-59$ month, $>11.5 \mathrm{~g} / \mathrm{dL}$ in $5-11$ years of age, $>12 \mathrm{~g} / \mathrm{dL}$ in 12 years of age) and normocytic normochromic cells. Exclusion criteria were: severe acute infection (pneumonia), chronic diseases (cardiac, renal, hepatic, autoimmune disease, immunodefi-ciencies), hematological disorders, chronic gastrointes-tinal diseases, already diagnosed celiac disease, bleeding, intake of any cytotoxic agents or radiotherapy in the last 6 weeks.

A detailed history with emphasis on current or past gastrointestinal symptoms was taken from the children or their parents. Nutritional status was assessed at the time of entry into the study. All eligible anemic children 
underwent hematological work-up that included hemogram, peripheral blood smear, red blood cell indices, serum iron levels, total iron binding capacity, transferrin saturation and serum ferritin levels. Stool examination was done on two consecutive days for identification of parasitic infestations. Children who were recruited as controls had a complete blood count done prior to enrolment.

We measured IgA-anti tissue transglutaminase (IgAtTG) by ELISA (Aeskulisa) in all included patients. IgAtTG levels $>18 \mathrm{U} / \mathrm{mL}$ were as considered positive as per information provided by the manufacturer, levels between 12 and $18 \mathrm{U} / \mathrm{mL}$ were considered as equivocal, and levels $<12 \mathrm{U} / \mathrm{mL}$ were considered as negative. All children who screened positive for celiac disease underwent upper gastrointestinal (UGI) endoscopy. Four biopsy specimens were taken from the second/first portion of the duodenum, out of which at least one sample was taken from the duodenal bulb. Histopathological examination of duodenal biopsies was performed by a histopathologist blinded to clinical history, and the result was graded using the modified Marsh grading [7]. Final diagnosis of CD was based on serology and endoscopic duodenal biopsy (Grade-3). Children diagnosed as CD were adviced strict gluten-free diet and oral iron supple-mentation for nutritional anemia. Children with IDA were advised oral iron preprations and dietary supplementation.

A sample size of 138 children with anemia (and 138 non-anemic controls) was calculated to determine the proportion of celiac disease with an estimated proportion of $10 \%$ [5] with absolute precision of 0.05 and confidence interval of $95 \%$. Assuming $10 \%$ loss to follow up, 152 cases and control were recruited. The statistical software SPSS 17 for Windows (Illinois, Chicago) was used for statistical analysis. Fischer's exact test was applied to compare the proportion IgA-tTG positive patients among cases and controls. Ethical clearance was obtained from Institutional ethical committee of University College of Medical Sciences. A written informed consent was obtained from parents of children eligible for inclusion, and assent was taken from children $\geq 7$ years of age.
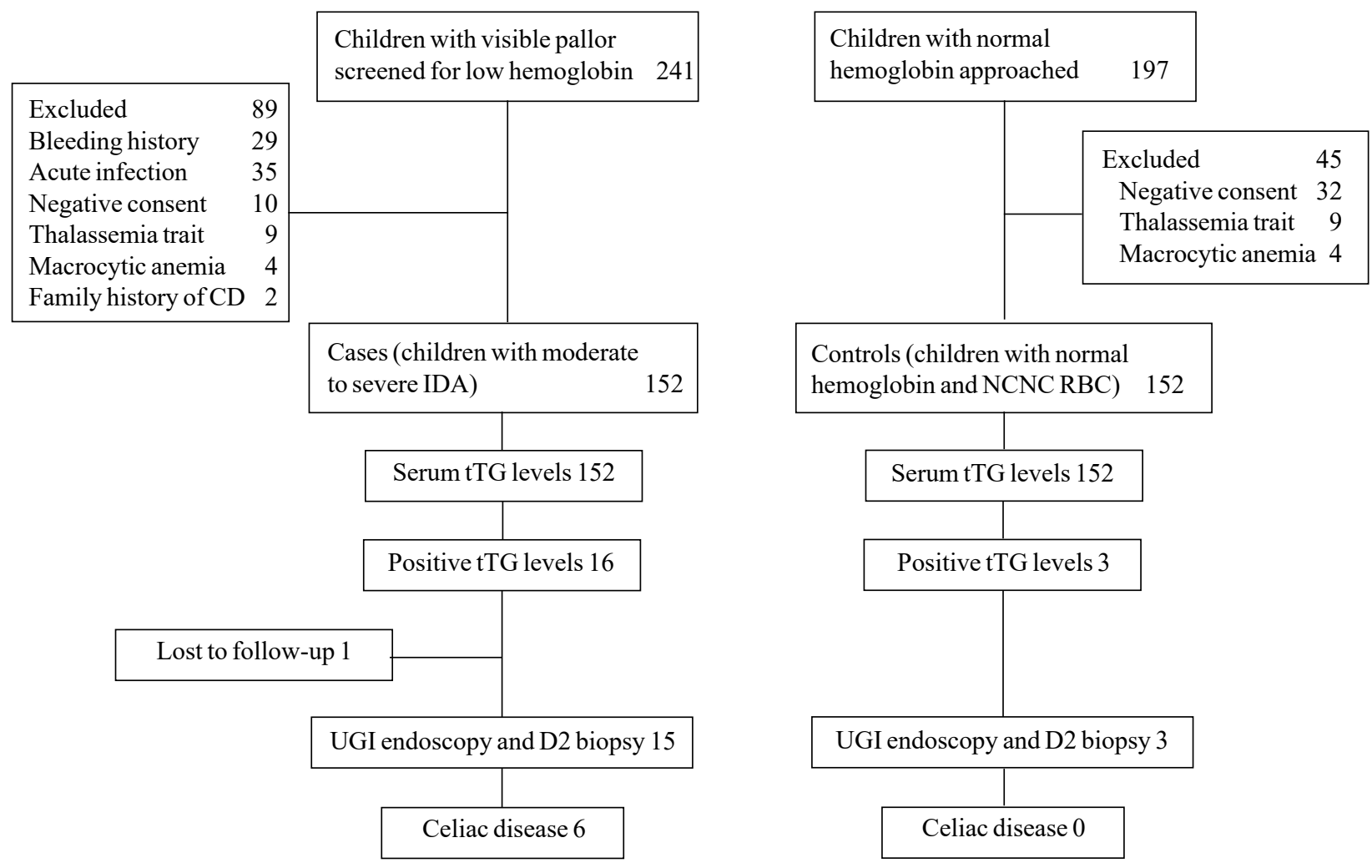

CD: celiac disease; IDA: iron deficiency anemia; NCNC RBC: normocytic normochromic red blood cells; $t$ TG: tissue transglutaminase; UGI: upper gastro-intestinal; D2: second part of duodenum.

FIG.1 Study flow chart. 
TABLE I DEMOGRAPHIC CHARACTERISTICS OF CHILDREN WITH AND WiTHOUT ANEMIA

\begin{tabular}{llr}
\hline & Cases $(n=152)$ & Controls $(n=152)$ \\
\hline Gender (M:F) & 1.33 & 1.23 \\
${ }^{\#}$ Age (mo) & $41(32.9)$ & $86.9(39.0)$ \\
WAZ & $-1.06(0.92)$ & $-0.93(0.96)$ \\
${ }^{\#} \mathrm{HAZ}$ & $-1.41(0.87)$ & $-1.12(0.95)$ \\
WHZ & $-0.47(1.36)$ & $-0.12(1.51)$ \\
MAC $(\mathrm{cm})$ & $15.20(1.63)$ & $16.63(1.85)$ \\
${ }^{*}$ Hemoglobin $(\mathrm{g} / \mathrm{dL})$ & $7.71(1.80)$ & $12.2(0.74)$ \\
\hline
\end{tabular}

All value in mean (SD); WAZ: weight for age $z$ score; HAZ: height for age $z$ score; WHZ: weight for height $z$ score; MAC: midarm circumference. ${ }^{*} P=0.001 ;{ }^{\#} P<0.01$.

\section{RESULTS}

The flow of participants in the study is shown in Fig.1. We included 152 cases and 152 controls. The non-anemic controls were of higher age group as compared to anemic cases [mean (SD): 86.9 (39) months vs 41 (32.9) months; $P=0.002]$ (Table I).

Increased prevalence of IgA-TTG was found among anemic children, as compared to non-anemic controls (16 vs $3 ; P=0.007)$. Endoscopy was conducted in 18 children (15 anemic), of which normal villous pattern was seen in 3 controls and 5 with anemia Marsh Grade I and grade IIIb findings were seen in 6 and 2, respectively of the anemic children. Two anemic children had giardiasis. After UGI endoscopy and biopsy, six (3.9\%) children with moderate or severe anemia and none of the control group were diagnosed as celiac disease $(P=0.013)$. Among 92 children with moderate anemia, one (1.08\%) patient had celiac disease while among 59 children with severe anemia $5(8.5 \%)$ had celiac disease. There was a significant difference between the mean (SD) hemoglobin level between patients with celiac disease and patients without celiac disease $[6.38(1.13) \mathrm{g} / \mathrm{dL} v s$ 7.77 (1.80) g/dL; $P=0.029]$. Subgroup analysis between anemic patients with or without celiac disease is mentioned in Table II.

\section{Discussion}

This cross-sectional study showed that celiac disease accounted for $8.5 \%$ and $3.9 \%$ of children with severe and moderate to severe iron deficiency anemia, respectively at
TABLE IICOMPARISON OF ANEMIC PATIENTS With OR WithouT Celiac Disease

\begin{tabular}{llr}
\hline & $\begin{array}{l}\text { Celiac disease } \\
(n=6)\end{array}$ & $\begin{array}{r}\text { No celiac disease } \\
(n=146)\end{array}$ \\
\hline Wasting & 0 & $16(11 \%)$ \\
Stunting & 0 & $30(20.5 \%)$ \\
\#Hemoglobin $(\mathrm{g} / \mathrm{dL})$ & $6.4(1.13)$ & $7.8(1.80)$ \\
*Severe anemia & $5(83.3 \%)$ & $54(37 \%)$ \\
Moderate anemia & $1(16.6 \%)$ & $92(63 \%)$ \\
${ }^{*} P=0.02 ;$ All value in no $(\%)$ & except ${ }^{*}$ hemoglobin in mean $(S D)$.
\end{tabular}

a tertiary-care hospital outpatient setting in Northern India.

Studies in children with iron-deficiency anemia are limited [1,8,9]. A study by Ertekin, et al. [9] among Turkish children had similar results with a mean hemoglobin level significantly lower in IDA patients with $\mathrm{CD}$ than in those without CD [9]. Moreover, those with severe anemia had higher odds of having CD.

As prevalence of anemia in India children 6 months59 months is $58.4 \%$ [2], there is a greater chance of missing those children with celiac disease presenting atypically as anemia. Early identification of these subclinical cases in childhood assumes greater importance since these patients are at risk of malignancy and mortality in later life and morbidity like the presence of unsuspected nutritional deficiencies.

The major limitation of this study is that being a hospital-based study results obtained in this study might not be representative of the whole population. The sample size was inadequate to find other clinical predictors of celiac disease in anemic children. Not doing IgA levels to screen for IgA deficiency, non-evaluation of occult blood loss in stool, and controls not being age matched are other study limitations. No loss to follow-up and outcome assessment using endoscopy are the strengths of the present study.

We conclude that anemic children, especially those presenting with severe anemia have significantly higher likelihood of having CD. Physicians treating children with severe anemia may consider screening them for celiac disease. We recommend community-based studies to confirm these findings.

\section{What ThIS StUdy Adds?}

- Severely anemic patients have higher chances of having associated celiac disease. 
Contributors: MN: Study conception and manuscript writing; RN: Data collection, analysis and manuscript writing; DS: Study conception, and critical review of manuscript for intellectual content; ASP, VM and MK: Study related procedures (data collection), their interpretation and critical inputs into manuscript.

Funding: None; Competing interests: None stated.

\section{REFERENCES}

1. Kavimandan A, Sharma M, Verma AK, Das P, Mishra P, Sinha S, et al. Prevalence of celiac disease in nutritional anemia at a tertiary care center. Indian J Gastroenterol. 2013;33:114-8.

2. National Family Health Survey-4, 2015-16: India Fact Sheet. Ministry of Health and Family Welfare. Available from: http://rchiips.org/NFHS/pdf/NFHS4/India.pdf. Accessed April 2, 2017.

3. Hill ID, Dirks MH, Liptak GS, Colletti RB, Fasano A, Guandalini S, et al. Guideline for the Diagnosis and Treatment of Celiac Disease in Children: Recommendations of the North American Society for Pediatric Gastroenterology, Hepatology. J Pediatr Gastroenterol Nutr. 2005;40:1-19.

4. World Health Organization: Department of Nutrition for
Health and development; Haemoglobin concentration for the Diagnosis of Anaemia and Assessment of Severity. Geneva, WHO, 2011. (WHO/NMH/NHD/MNM/11.1). Available from: http://apps.who.int/iris/bitstream/ 10665/101179/1/WHO_NMH_NHD_EPG_14.1_eng. pdf. Accessed December 27, 2016.

5. World Health Organization: Serum Ferritin Concentrations for the Assessment of Iron Status and Iron Deficiency in Populations. Vitamin and Mineral Nutrition Information System. Geneva, World Health Organization, 2011. (WHO/NMH/NHD/MNM/11.2). Available from: http://www.who.int/vmnis/indicators/serum_ferritin.pdf. Accessed December 27, 2016.

6. Bainton DF, Finch CA. The diagnosis of iron deficiency anemia. Am J Med. 1964;37:62-70.

7. Marsh MN, Crowe PT. Morphology of the mucosal lesion in gluten sensitivity. Baillieres Clin Gastroenterol. 1995;9:273-93.

8. Kalayci AG, Kanber Y, Birinci A, Yildiz L, Albayrak D. The prevalence of coeliac disease as detected by screening in children with iron deficiency anaemia. Acta Paediatr. 2005;94:678-81.

9. Ertekin V, Tozun MS, Küçük N. The prevalence of celiac disease in children with iron-deficiency anemia. Turk J Gastroenterol. 2013;24:334-8. 